\title{
Efficacy of arterial spin labeling for detection of the ruptured micro-arteriovenous malformation: illustrative cases
}

\author{
Ryuzaburo Kochi, MD, PhD, ${ }^{1}$ Hidenori Endo, MD, PhD, ${ }^{1,2}$ Hiroki Uchida, MD, PhD, ${ }^{1}$ Tomohiro Kawaguchi, MD, PhD, ${ }^{1}$ \\ Shunsuke Omodaka, MD, PhD, ${ }^{3}$ Yasushi Matsumoto, $\mathrm{MD},{ }^{3}$ and Teiji Tominaga, MD, $\mathrm{PhD}^{4}$ \\ Departments of ${ }^{1}$ Neurosurgery and ${ }^{3}$ Neuroendovascular Therapy, Kohnan Hospital, Sendai, Miyagi, Japan; ${ }^{2}$ Division of Advanced Cerebrovascular Surgery, Tohoku University \\ Graduate School of Medicine, Sendai, Miyagi, Japan; and ${ }^{4}$ Department of Neurosurgery, Tohoku University Graduate School of Medicine, Sendai, Miyagi, Japan
}

\begin{abstract}
BACKGROUND Diagnosis of a microarteriovenous malformation (micro-AVM) is difficult, especially in the acute stage of rupture because of the small size of the nidus and the existence of hematoma. We report two cases of ruptured micro-AVMs detected by arterial spin labeling (ASL).

OBSERVATIONS In one case, a 45-year-old male was transported with a complaint of right hemiparesis. Computed tomography (CT) revealed a right parietal lobar hemorrhage. Standard magnetic resonance imaging (MRI) showed no abnormal findings as the cause of the hemorrhage. ASL 23 days after the onset demonstrated high signals on the medial wall of the hematoma. Digital subtraction angiography (DSA) showed a micro-AVM in accordance with the site of high signals on ASL. In another case, a 38-year-old female was transported with a complaint of left hemianopsia. CT on admission revealed a right parietal lobar hemorrhage. Standard MRI showed no abnormal findings as the cause of the hemorrhage. ASL 15 days after the onset demonstrated high signals on the internal wall of the hematoma. DSA showed micro-AVM in accordance with the site of high signaling on ASL. Both cases were successfully treated with open surgery.
\end{abstract}

LESSONS ASL can manifest micro-AVMs as high signals within the hematoma. ASL is a useful less-invasive screening tool for the detection of ruptured micro-AVMs.

https://thejns.org/doi/abs/10.3171/CASE21597

KEYWORDS arterial spin labeling; micro-arteriovenous malformation; intracerebral hematoma; surgery

Arterial spin labeling (ASL) has emerged as a magnetic resonance imaging (MRI) technique that can be used to evaluate cerebral blood flow (CBF). It can visualize CBF using magnetically labeled protons in arterial blood as an endogenous tracer. ASL is advantageous because it is noninvasive and can be performed without the administration of a nuclear tracer or contrast media. ASL has been reported to be effective as a screening tool to diagnose cerebral ischemia, epilepsy, and hyperperfusion syndrome. ${ }^{1-5}$

An intracranial arteriovenous malformation (AVM) is one of the potential causes of intracranial hemorrhaging in children or young adults. Digital subtraction angiography (DSA) is the gold standard for the definitive diagnosis of AVMs. MRI is a less invasive screening tool that can be used to diagnose AVMs, which are detected as signal flow voids within a hematoma in the acute stage of rupture. However, it is sometimes difficult to diagnose an AVM especially when the nidus is small. Here, we report two cases of ruptured micro-AVMs successfully detected with ASL.

\section{Illustrative Cases}

\section{Case 1}

A 45-year-old male was transported as an emergency to our hospital with sudden onset of right hemiparesis, dysarthria, and motor aphasia. Computed tomography (CT) on admission revealed a right parietal lobar hemorrhage (Fig. 1A). MRI was also conducted on the same day, but magnetic resonance angiography (MRA) and

ABBREVIATIONS ASL = arterial spin labeling; $\mathrm{AVM}=$ arteriovenous malformation; $\mathrm{CBF}=$ cerebral blood flow; $\mathrm{CT}=$ computed tomography; $\mathrm{DSA}=$ digital subtraction angiography; DAVF = dural arteriovenous fistula; MRA = magnetic resonance angiography; $\mathrm{MRI}=$ magnetic resonance imaging; $\mathrm{T} 2 \mathrm{Wl}=\mathrm{T} 2$-weighted image; TOF = time of flight. 

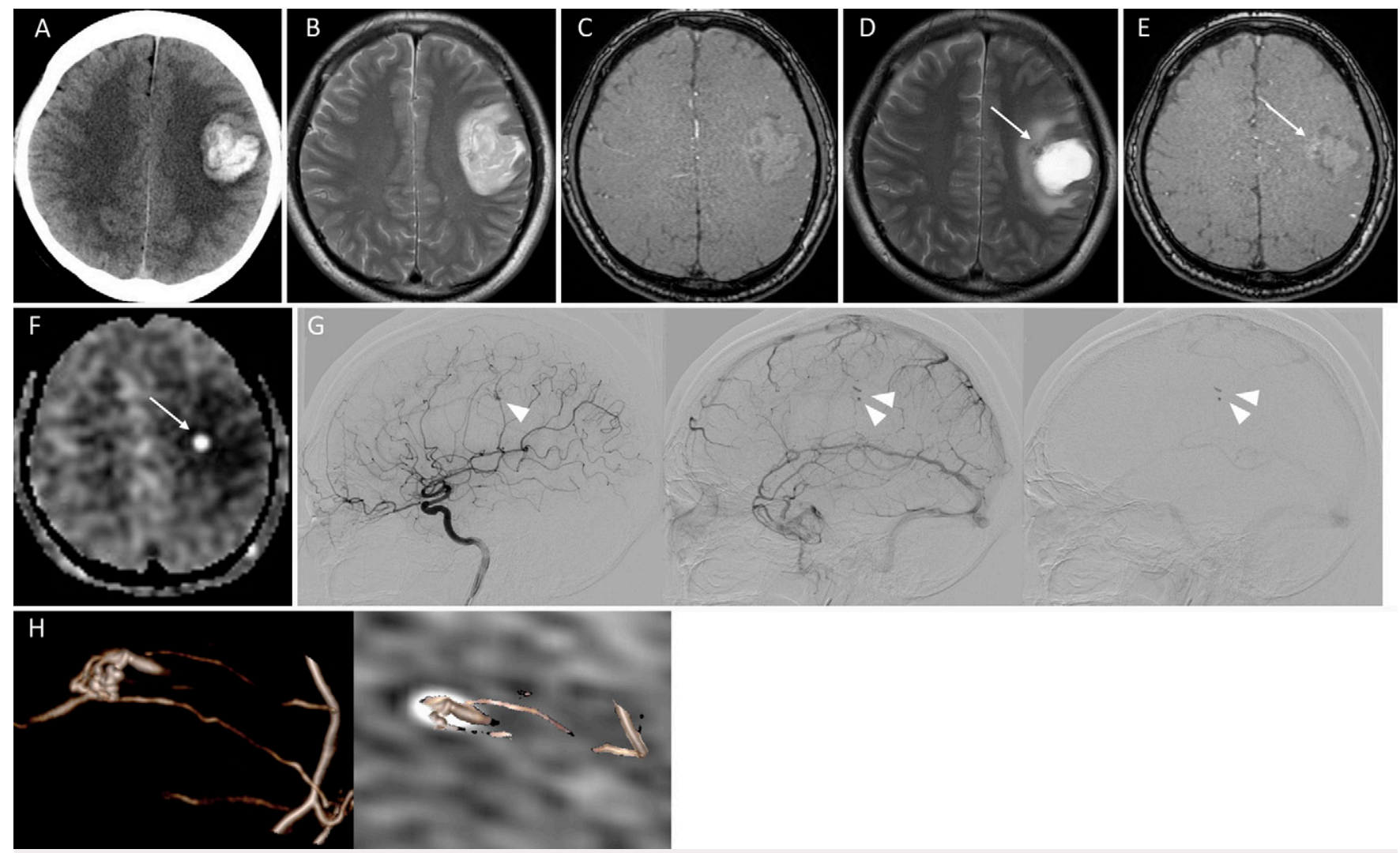

FIG. 1. A: CT on admission showing the left parietal hemorrhage. B and C: T2WI (B) and TOF-MRA (C) on admission showing no abnormal vascular structure within the hematoma. D: T2WI 23 days after the hemorrhage showing a hematoma with an adjacent small low-intensity lesion (arrow). E: TOFMRA showing slight high intensity signals at the site of a T2 low-intensity lesion (arrow). F: ASL showing clear high signal at the site of a T2 low-intensity lesion (arrow). G: Digital subtraction angiography (left, arterial phase; middle, venous phase; right, late venous phase) showing the nidus in the arterial phase (arrowhead) and the pooled contrast media within the nidus and part of the drainage system until the late venous phase (double arrowhead). $\mathrm{H}$ : Three-dimensional (3D) angiography (left) and a fusion image of the 3D angiography and the ASL images (right) showing the spatial correspondence of the site of the nidus and the ASL high intensity.

T2-weighted image (T2WI) showed no remarkable findings indicating the cause of the hemorrhage (Fig. $1 \mathrm{~B}$ and $\mathrm{C}$ ). We treated the patient conservatively with blood pressure management and administration of glycerol. A second MRI was performed 23 days after the onset. T2WI and MRA showed subtle abnormal findings on the medial wall of the hematoma, but the image was not clear enough to support the existence of abnormal vessels (Fig. 1D and E). On the other hand, ASL demonstrated clear dotted high signals on the medial wall of the hematoma cavity (Fig. 1F), which facilitated the use of angiography. DSA revealed a micro-AVM in accordance with the site of ASL high signaling (Fig. 1G). The AVM was composed of a $7 \mathrm{~mm}$ nidus with two feeding arteries that were the branches of the left anterior parietal artery. A draining vein arose from the medial side of the nidus and flew toward the internal cerebral vein. Contrast media pooled within the nidus until the late venous phase. A fusion image of the DSA and the ASL CBF maps clearly demonstrated the spatial correspondence of the nidus and the ASL high signal lesion (Fig. 1H). The AVM was removed 32 days after the onset of the rupture, and the intraoperative DSA showed no residual shunt (not shown). The ASL high signal disappeared with MRI 8 days after the operation. The patient was discharged to a rehabilitation hospital 15 days after the operation without additional neurological deficits.

\section{Case 2}

A 38-year-old female was transported as an emergency to our hospital with left hemihypoesthesia and left hemispatial neglect. CT revealed a right parietal lobar hemorrhage (Fig. 2A). MRA and T2WI performed on the same day showed no remarkable findings suggesting the cause of the hemorrhage (Fig. 2B and $\mathrm{C}$ ). The patient was medically treated in the acute phase. Follow-up T2WI 15 days after onset showed no apparent source of bleeding (Fig. 2D). MRA showed subtle abnormal findings in the lateral aspect of the hematoma, which was not clear enough to support the existence of abnormal vessels (Fig. 2E). On the other hand, ASL demonstrated clear dotted high signaling at the site of MRA high intensity (Fig. 2F), which facilitated the use of angiography. The AVM was composed of a $7.4 \mathrm{~mm}$ nidus that was fed by the right temporoparietal artery (Fig. 2G). A draining vein arose from the lateral side of the nidus, which drained toward the superior sagittal sinus. Contrast media was pooled within the nidus until the late venous phase. A fusion image of DSA and ASL clearly demonstrated the spatial correspondence of the nidus and the ASL high signals (Fig. 2H). Successful AVM removal was achieved 30 days after the onset of the rupture, and intraoperative DSA showed no residual shunt. ASL high signals disappeared with MRI 10 days after the operation. The patient was discharged to a rehabilitation hospital 17 days after the operation without additional neurological deficits. 

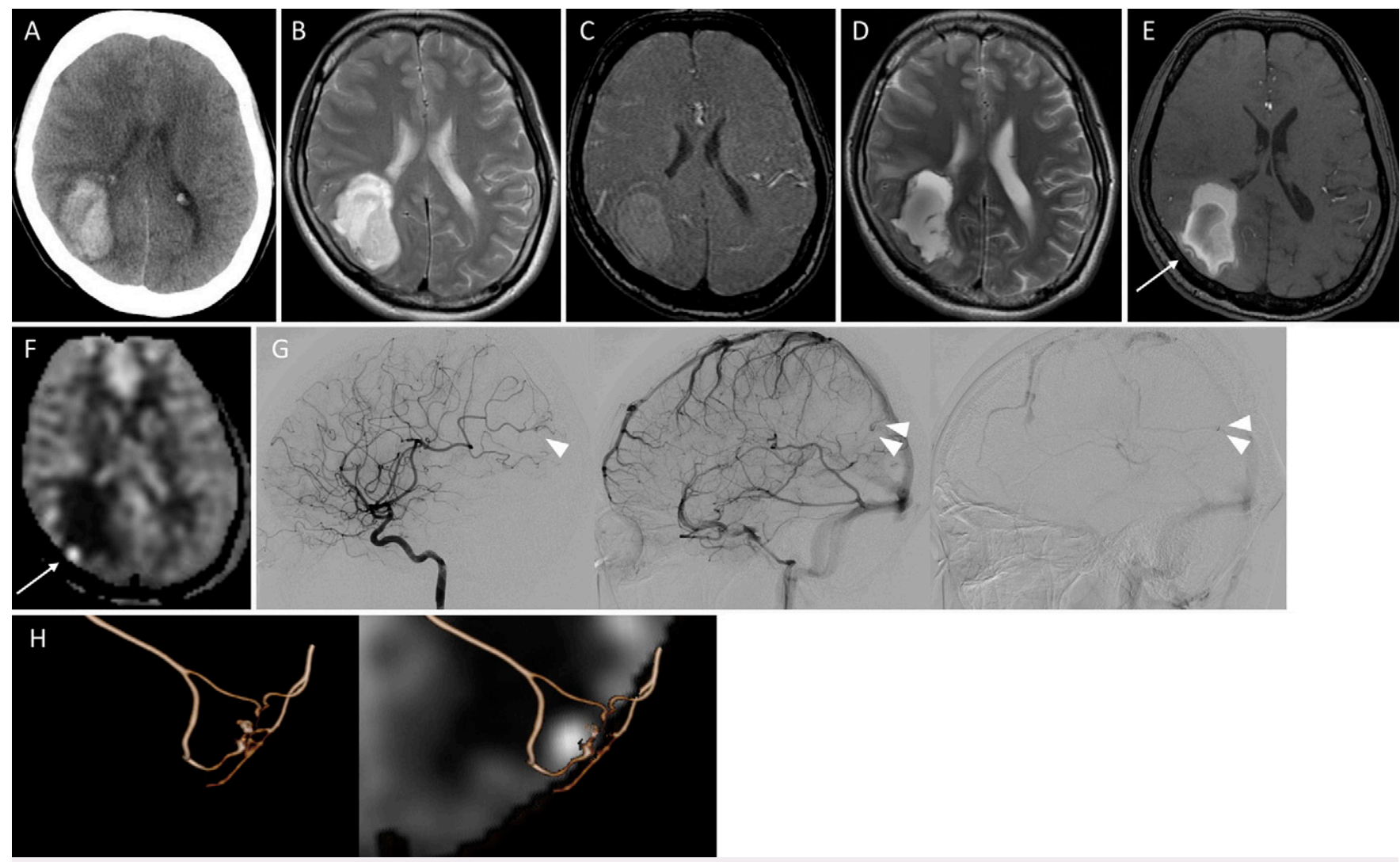

FIG. 2. A: CT on admission showing the right parietal hemorrhage. B and C: T2WI (B) and TOF-MRA (C) on admission showing no abnormal vascular structure within the hematoma. D: T2WI 15 days after the hemorrhage showing a hematoma without an obvious source of bleeding. E: TOF-MRA showing slight high intensity signals at the lateral margin of the hematoma (arrow). F: ASL showing a clear high signal at the site of the TOF high intensity lesion. G: DSA (left, arterial phase; middle, venous phase; right, late venous phase) showing the nidus in the arterial phase (arrowhead) and the pooled contrast media within the nidus until the late venous phase (double arrowhead). H: Three-dimensional (3D) angiography (left) and the fusion image of the 3D angiography and the ASL images (right) showing the spatial correspondence of the site of the nidus and the ASL high intensity.

\section{Discussion}

Micro-AVMs are defined as a subgroup of pial AVMs characterized by a nidus of $1 \mathrm{~cm}$ or smaller. ${ }^{6}$ The incidence of micro-AVMs was reported to be $8 \%-10.7 \%$ of surgically treated AVMs. ${ }^{7,8}$ Micro-AVMs typically tend to cause large intracranial hemorrhages, resulting in significant neurological deficits in young adults. ${ }^{8}$ Early angiography may misdiagnose ruptured micro-AVMs with large hematomas. Slow flow within the nidus, intralesional thrombosis, posthemorrhagic vascular spasm, or compression of the nidus by the hematoma may account for the lack of visualization of a micro-AVM in the acute stage of rupture. ${ }^{9,10}$ If initial angiography is negative or dubious, repetition of DSA and superselective angiography are recommended ${ }^{8,11}$ but these have some invasive aspects, such as radiation exposure, catheter use, and contrast media injection. MRI is a less-invasive screening tool that can be used to detect AVMs before DSA. However, micro-AVMs are also difficult to diagnose with standard MRI protocols especially in the acute phase of hemorrhaging because of their small size and the existence of the surrounding brain edema and the hematoma. ${ }^{12}$ Time-of-flight (TOF) MRA is a T1-based technique that utilizes inflow effect and is clinically useful to visualize vascular structure. Blood flow is shown as hyperintense signal in TOF, which could be vague within the intracerebral hematoma. ${ }^{13}$ This could be disadvantageous to diagnose ruptured micro-AVMs. From the results of this study, ASL can be a useful screening tool to detect ruptured micro-AVMs before a definitive diagnosis with DSA, especially when the patients are children or young adults.

\section{Observations}

We report the efficacy of ASL for detecting ruptured micro-AVMs that are not obvious in the other magnetic resonance sequences. In ASL, arterial blood is magnetically labeled at the cervical segment, and brain images are acquired following a short delay to allow the labeled arterial blood to flow through the cerebral arteries and into the capillary bed..$^{14,15}$ Under normal conditions, the labeled blood is not detected within the venous structures as the capillary transit time is longer than the T1 delay time of the labeled arterial spins. ${ }^{14,15}$ In the presence of an arteriovenous shunt, such as an AVM and dural arteriovenous fistula (DAVF), the transit time of the labeled blood is shortened due to the lack of a capillary bed. Consequently, ASL signals can be detected within the draining system, which can suggest the diagnosis of an intracranial arteriovenous shunt. ${ }^{16,17}$ This venous signal on ASL was reported useful for the diagnosis of DAVF or small AVM, and the diagnostic sensitivity was as high as $78 \% .^{9}$ In addition, Heit et al..$^{18}$ reported the utility of venous ASL signals for the diagnosis of residual AVM following 
stereotactic radiosurgery. In the present cases, ASL high signals were observed within the nidus in addition to part of the draining system. Fusion images from ASL and DSA clearly showed the spatial correspondence of the sites of the micro-AVMs and the ASL high signals. ASL high signals within the nidus are due to pooled blood within the nidus. In ASL for cerebral aneurysms, the pooled blood within the aneurysms can be detected by the ASL as trapped labeled spin within the aneurysm. ${ }^{19}$ This ASL high signal was reported to be useful for detecting residual filling in cerebral aneurysms after treatment with flow diverter. ${ }^{19}$ In our cases, ASL high signaling might reflect occlusion of the drainage system and subsequent pooled blood within the nidus, which facilitates rupture of the AVM. One of the conclusive mechanisms of AVM rupture is increased resistance in the drainage system. ${ }^{20}$ Thus, this ASL high signal within the nidus might be an imaging feature of ruptured micro-AVM. We did not perform ASL in the initial MRI in the acute stage of bleeding, which is a limitation of this study. If early ASL can demonstrate the existence of AVM in the acute stage with high sensitivity, it would be informative to avoid invasive examinations.

\section{Lessons}

ASL can demonstrate micro-AVMs as high signals within the hematoma. ASL is a useful and less-invasive screening tool for the detection of ruptured micro-AVMs.

\section{References}

1. Detre JA, Alsop DC, Vives LR, Maccotta L, Teener JW, Raps EC. Noninvasive MRI evaluation of cerebral blood flow in cerebrovascular disease. Neurology. 1998;50(3):633-641.

2. Shimogawa T, Morioka T, Sayama T, et al. The initial use of arterial spin labeling perfusion and diffusion-weighted magnetic resonance images in the diagnosis of nonconvulsive partial status epileptics. Epilepsy Res. 2017;129:162-173.

3. Deibler AR, Pollock JM, Kraft RA, Tan H, Burdette JH, Maldjian JA. Arterial spin-labeling in routine clinical practice, part 1: technique and artifacts. AJNR Am J Neuroradiol. 2008;29(7):1228-1234.

4. Deibler AR, Pollock JM, Kraft RA, Tan H, Burdette JH, Maldjian JA. Arterial spin-labeling in routine clinical practice, part 2: hypoperfusion patterns. AJNR Am J Neuroradiol. 2008;29(7):1235-1241.

5. Deibler AR, Pollock JM, Kraft RA, Tan H, Burdette JH, Maldjian JA. Arterial spin-labeling in routine clinical practice, part 3: hyperperfusion patterns. AJNR Am J Neuroradiol. 2008;29(8):1428-1435.

6. Yasargil MG. Pathological considerations. In: Microneurosurgery IIIA. AVM of the Brain, History, Embryology, Pathological Considerations, Hemodynamics, Diagnostic Studies, Microsurgical Anatomy. Thieme; 1987:85-91.

7. Stiver SI, Ogilvy CS. Micro-arteriovenous malformations: significant hemorrhage from small arteriovenous shunts. Neurosurgery. 2000;46(4):811-819.

8. Alén JF, Lagares A, Paredes I, et al. Cerebral microarteriovenous malformations: a series of 28 cases. J Neurosurg. 2013;119(3): 594-602.

9. Le TT, Fischbein NJ, André JB, Wijman C, Rosenberg J, Zaharchuk G. Identification of venous signal on arterial spin labeling improves diagnosis of dural arteriovenous fistulas and small arteriovenous malformations. AJNR Am J Neuroradiol. 2012;33(1):61-68.
10. Hino A, Fujimoto M, Yamaki T, Iwamoto Y, Katsumori T. Value of repeat angiography in patients with spontaneous subcortical hemorrhage. Stroke. 1998;29(12):2517-2521.

11. Ogilvy CS, Heros RC, Ojemann RG, New PF. Angiographically occult arteriovenous malformations. J Neurosurg. 1988;69(3): $350-355$.

12. Willinsky R, TerBrugge $K$, Montanera W, Wallace $C$, Aggarwal $S$. Micro-arteriovenous malformations of the brain: superselective angiography in diagnosis and treatment. AJNR Am J Neuroradiol. 1992;13(1):325-330.

13. Warren DJ, Hoggard N, Walton L, et al. Cerebral arteriovenous malformations: comparison of novel magnetic resonance angiographic techniques and conventional catheter angiography. Neurosurgery. 2001;48(5):973-983.

14. Telischak NA, Detre JA, Zaharchuk G. Arterial spin labeling MRI: clinical applications in the brain. J Magn Reson Imaging. 2015; 41(5):1165-1180.

15. Zaharchuk $\mathrm{G}$. Arterial spin-labeled perfusion imaging in acute ischemic stroke. Stroke. 2014;45(4):1202-1207.

16. Hodel J, Leclerc X, Kalsoum E, et al. Intracranial arteriovenous shunting: detection with arterial spin-labeling and susceptibilityweighted imaging combined. AJNR Am J Neuroradiol. 2017;38(1): 71-76.

17. Wolf RL, Wang J, Detre JA, Zager EL, Hurst RW. Arteriovenous shunt visualization in arteriovenous malformations with arterial spinlabeling MR imaging. AJNR Am J Neuroradiol. 2008;29(4):681-687.

18. Heit JJ, Thakur NH, Iv M, et al. Arterial-spin labeling MRI identifies residual cerebral arteriovenous malformation following stereotactic radiosurgery treatment. J Neuroradiol. 2020;47(1):13-19.

19. Kulanthaivelu K, Peer S, Biswas S, et al. "Trapped labelled spins"related signal on arterial spin labelling in the assessment of flowdiverted aneurysms: preliminary experience. Neuroradiology. Published online June 14, 2021. doi: 10.1007/s00234-021-02721-y.

20. Hademenos GJ, Massoud TF. Risk of intracranial arteriovenous malformation rupture due to venous drainage impairment. A theoretical analysis. Stroke. 1996;27(6):1072-1083.

\section{Disclosures}

Dr. Matsumoto reported personal fees from Stryker Japan, personal fees from Medtronic Japan, personal fees from Kaneka medics, personal fees from Takeda Pharmaceutical Limited, personal fees from GE healthcare, personal fees from Medico's Hirata, personal fees from Century medical, personal fees from Fuji systems, and personal fees from Otuka Pharmaceutical Limited outside the submitted work. Dr. Tominaga reported grants from Ministry of Education, Culture, Sports, Science and Technology outside the submitted work. No other disclosures were reported.

\section{Author Contributions}

Conception and design: Endo, Kochi, Omodaka. Acquisition of data: Endo, Kochi, Uchida, Matsumoto. Analysis and interpretation of data: Endo, Kochi. Drafting the article: Kochi, Kawaguchi, Omodaka. Critically revising the article: Endo. Reviewed submitted version of manuscript: Endo, Omodaka. Approved the final version of the manuscript on behalf of all authors: Endo. Study supervision: Matsumoto, Tominaga.

\section{Correspondence}

Hidenori Endo: Kohnan Hospital, Miyagi, Japan. h-endo@nsg.med. tohoku.ac.jp. 